\title{
Spontaneous Appearance of the Spin-Triplet Fulde-Ferrell-Larkin-Ovchinnikov Phase in a Two-Band Model: Possible Application to $\mathrm{LaFeAsO}_{1-x} \mathrm{~F}_{x}$
}

\author{
M. Zegrodnik • J. Spałek
}

Received: 16 June 2014 / Accepted: 17 September 2014 / Published online: 25 October 2014

(C) The Author(s) 2014. This article is published with open access at SpringerLink.com

\begin{abstract}
The possibility of a spontaneous spin-triplet paired phase of the Fulde-Ferrell-Larkin-Ovchinnikov type is studied. As it is shown in a system with the dominant interband pairing and two distinct Fermi surface sheets, the Fermi wave-vector mismatch can be compensated by a nonzero center-of-mass momentum of the Cooper pairs. This idea is examined with the use of a model which describes the two hole-like bands in the iron-based superconductor. It is shown that for the proper range of model parameters, the minima of the free energy appear which correspond to a nonzero Cooper pair momentum. Different superconducting gap symmetries are analyzed, and the corresponding phase diagrams are shown.
\end{abstract}

Keywords Unconventional superconductivity · FFLO phase $\cdot$ Iron pnictides

\section{Introduction}

The so-called Fulde-Ferrel-Larkin-Ovchinnikov (FFLO) phase has been proposed decades ago $[1,2]$ and attracted much attention over the years. This unconventional superconducting phase can be induced by the external magnetic field in systems with high Maki parameter [3] for the case

M. Zegrodnik $(\bowtie) \cdot$ J. Spałek

Academic Centre for Materials and Nanotechnology, AGH

University of Science and Technology, Al. Mickiewicza 30, 30-059 Krakow, Poland

e-mail: michal.zegrodnik@agh.edu.pl

J. Spałek

Marian Smoluchowski Institute of Physics, Jagiellonian

University, ul. Reymonta 4, 30-059 Krakow, Poland

e-mail: ufspalek@if.uj.edu.pl of spin-singlet pairing. The Fermi wave-vector mismatch which appears in such conditions can be compensated by a nonzero center-of-mass momentum of the Cooper pairs. Experimental signs of the FFLO phase have been reported in the heavy fermion compound $\mathrm{CeCoIn}_{5}$ [4-6], as well as in organic superconductors [7-11]. Also, an indirect evidence of a superfluid FFLO phase in a system of ultracold atomic gas trapped in an array of one-dimensional tubes has been reported [12].

It has been proposed by us recently (M. Zegrodnik and J. Spałek, 2014, A spontaneous paired state with nonzero Cooper-pair momentum: Possible application to iron pnictides, unpublished) that a paired phase with nonzero Cooper pair momentum can appear in the absence of an external magnetic field in systems with dominant interband pairing and two distinct Fermi surface sheets. The high value of the Maki parameter would not be required for the formation of such phase. However, the electronic structure of the system at hand should exhibit certain features to create favorable conditions for nonzero momentum pairing. To study this idea, we use the interband spin-triplet pairing mechanism [13, 14] suggested for iron pnictides in [15]. It should be noted that with respect to the ironbased superconductors both spin-singlet [16-19] and spintriplet $[15,19,20]$, gap symmetries have been considered. In this work, we use the tight binding model which reflects the two hole-like bands of the iron-based compound $\mathrm{LaFeAsO}_{1-x} \mathrm{~F}_{x}$. The stability of the proposed phase against both the normal and the homogeneous paired phases is analyzed. Different symmetries of the superconducting gap are considered, and their influence on the properties of the nonzero momentum pairing is studied. One should note that our approach could be applied to other multiband systems with either spin-singlet or spin-triplet types of pairing. 


\section{Theoretical Model}

Following Raghu et al. [21], we use the tight binding model which describes the electronic structure of the Fe-As layer of $\mathrm{LaFeAsO}_{1-x} \mathrm{~F}_{x}$. However, we limit to the two holelike bands only. Additionally, spin-triplet pairing term is added (similarly as in [15]). The model Hamiltonian has the following form:

$\hat{\mathcal{H}}=\sum_{\mathbf{k} l \sigma}\left(E_{\mathbf{k} l}-\mu\right) \hat{n}_{\mathbf{k} l \sigma}-\frac{2}{N} \sum_{\mathbf{k} \mathbf{k}^{\prime} \mathbf{Q} m} J_{\mathbf{k}-\mathbf{k}^{\prime}} \hat{A}_{\mathbf{k}^{\prime} m \mathbf{Q}}^{\dagger} \hat{A}_{\mathbf{k} m \mathbf{Q}}$

where $l=1,2$ labels the bands, $\mu$ is the chemical potential, $N$ is the number of $\mathrm{Fe}$ atoms in the lattice, and $E_{\mathbf{k} l}$ are the dispersion relations which are plotted in Fig. 1b. One should note that the summations in the Hamiltonian are over the folded Brillouin zone which is marked by the solid line in Fig. 1a (for details of the folding procedure, see [21]). The second term in (1) is responsible for the interband spin-triplet pairing with the possibility of nonzero total momentum of the Cooper pairs, $\mathbf{Q}$. An analogical term has been introduced in [15] but for the two electron-like bands of the iron-based superconductor and without the inclusion of the nonzero momentum pairing. The spin-triplet pairing operators are defined as follows:

$$
\hat{A}_{\mathbf{k}, m \mathbf{Q}}^{\dagger} \equiv\left\{\begin{aligned}
\hat{c}_{\mathbf{k} \uparrow \uparrow}^{\dagger} \hat{c}_{-\mathbf{k}+\mathbf{Q} 2 \uparrow}^{\dagger} & m=1, \\
\hat{c}_{\mathbf{k} 1 \downarrow}^{\dagger} \hat{c}_{-\mathbf{k}+\mathbf{Q} 2 \downarrow}^{\dagger} \hat{q}^{\dagger} & m=-1, \\
\frac{1}{\sqrt{2}}\left(\hat{c}_{\mathbf{k} 1 \uparrow}^{\dagger} \hat{c}_{-\mathbf{k}+\mathbf{Q} 2 \downarrow}^{\dagger}+\hat{c}_{\mathbf{k} 1 \downarrow}^{\dagger} \hat{c}_{-\mathbf{k}+\mathbf{Q} 2 \uparrow}\right) & m=0 .
\end{aligned}\right.
$$

The second term in (1) is associated with the pairing mechanism induced by Hund's rule [13, 14]. As the Hund's coupling operates on particles from different bands, the resultant pairing has an interband character. Such approach results in spin-triplet, band-singlet paired phase with a symmetric gap parameter (even parity, e.g., s-wave, extended $\mathrm{s}$-wave, $\mathrm{d}$-wave). In the absence of magnetic ordering, one can focus on the superconducting A phase (equal spin) in which the Cooper pairs are in the states corresponding to $m= \pm 1$ only and $\Delta_{\mathbf{k}, 1 \mathbf{Q}}=\Delta_{\mathbf{k},-1 \mathbf{Q}} \equiv \Delta_{\mathbf{k} \mathbf{Q}}$ is fulfilled, where

$\Delta_{\mathbf{k}, \pm 1 \mathbf{Q}}=-\frac{2}{N} \sum_{\mathbf{k}^{\prime}} J_{\mathbf{k}-\mathbf{k}^{\prime}}\left\langle\hat{A}_{\mathbf{k}^{\prime}, \pm 1 \mathbf{Q}}\right\rangle$,

is the gap parameter corresponding to spin-up and spindown Cooper pairs. This phase is equivalent to the one corresponding to the following relations: $\Delta_{\mathbf{k}, 1 \mathbf{Q}}=\Delta_{\mathbf{k},-1 \mathbf{Q}} \equiv$ $0, \Delta_{\mathbf{k}, 0 \mathbf{Q}} \neq 0$, where

$\Delta_{\mathbf{k}, 0 \mathbf{Q}}=-\frac{2}{\sqrt{2} N} \sum_{\mathbf{k}^{\prime}} J_{\mathbf{k}-\mathbf{k}^{\prime}}\left\langle\hat{A}_{\mathbf{k}^{\prime}, 0 \mathbf{Q}}\right\rangle$,

is the gap parameter. For simplicity, we have assumed that in the superconducting state, all the pairs have the same total momentum $\mathbf{Q}$ (the Fulde-Ferrell phase) and we take $J_{\mathbf{k}}$ in the following form:

$J_{\mathbf{k}}=J_{0}+J_{1}\left(\cos k_{x}+\cos k_{y}\right)$,

where $J_{0}$ and $J_{1}$ determine the pairing strength. Such form of $J_{\mathbf{k}}$ has also been chosen in [15]. By using the mean field (Bardeen-Cooper-Schrieffer, BCS) approximation, one obtains the following form of the effective Hamiltonian:

$$
\begin{aligned}
& \hat{\mathcal{H}}_{H F}= \\
& \sum_{\mathbf{k} l \sigma}\left(E_{\mathbf{k} l}-\mu\right) \hat{n}_{\mathbf{k} l \sigma}+\sum_{\mathbf{k}, m= \pm 1}\left(\Delta_{\mathbf{k} \mathbf{Q}} \hat{A}_{\mathbf{k} m \mathbf{Q}}^{\dagger}+H . C .\right) \\
& \quad+\frac{N\left(\Delta_{\mathbf{Q}}^{(0)}\right)^{2}}{J_{0}}+\frac{2 N\left(\Delta_{\mathbf{Q}}^{(1)}\right)^{2}}{J_{1}}
\end{aligned}
$$

where the gap is a mixture of s-wave and extended s-wave gap symmetries, i.e.,

$\Delta_{\mathbf{k} \mathbf{Q}}=\Delta_{\mathbf{Q}}^{(0)}+\Delta_{\mathbf{Q}}^{(1)}\left(\cos k_{x}+\cos k_{y}\right)$.

However, other gap symmetries can also be analyzed, e.g., the d-wave:

$\Delta_{\mathbf{k} \mathbf{Q}}=\Delta_{\mathbf{Q}}^{(1)}\left(\cos k_{x}-\cos k_{y}\right)$.

The amplitudes $\Delta^{(0)}, \Delta^{(1)}$ and the chemical potential are calculated by solving the set of self-consistent equations
Fig. 1 Two hole-like Fermi surface sheets in the folded Brillouin zone (a) and the electronic structure (b) for band filling $n=1.78838$. The energies are normalized to the bare bandwidth $W$. Note the Fermi wave-vector mismatch $\Delta k$ between the states $\left(\mathbf{k}=\mathbf{k}_{F}, l=1\right)$ and

$\left(\mathbf{k}=-\mathbf{k}_{F}, l=2\right)$
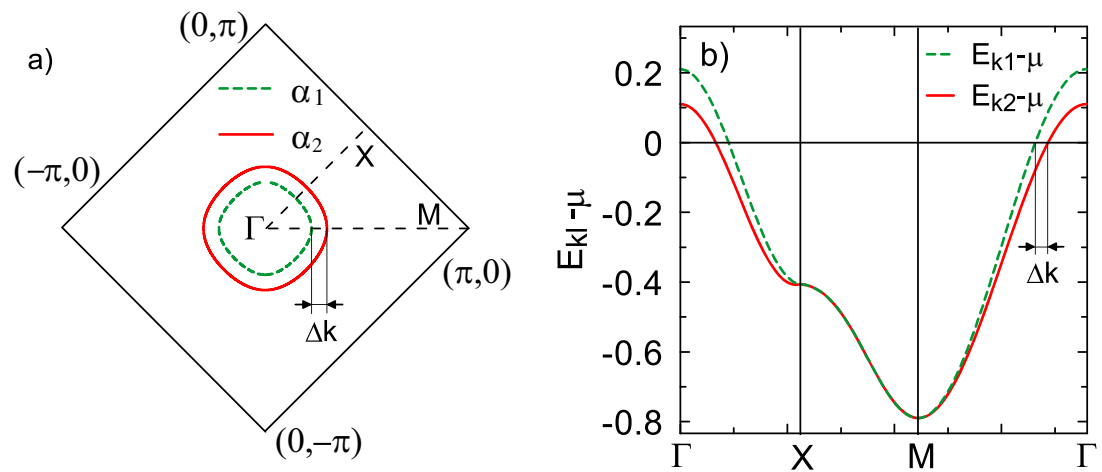
numerically, whereas the vector $\mathbf{Q}$ is determined by minimizing the free energy of the system.

It should be noted that with respect to the considered tight binding model, the even-parity pairing can be also analyzed within the spin-singlet, band-triplet channel. The corresponding Hamiltonian has the following form:

$\hat{\mathcal{H}}^{s}=\sum_{\mathbf{k} l \sigma}\left(E_{\mathbf{k} l}-\mu\right) \hat{n}_{\mathbf{k} l \sigma}-\frac{2}{N} \sum_{\mathbf{k} \mathbf{k}^{\prime}} J_{\mathbf{k}-\mathbf{k}^{\prime}} \hat{B}_{\mathbf{k}^{\prime} \mathbf{Q}}^{\dagger} \hat{B}_{\mathbf{k} \mathbf{Q}}$,

where the spin-singlet pairing operator has been introduced:

$\hat{B}_{\mathbf{k} \mathbf{Q}}^{\dagger}=\frac{1}{\sqrt{2}}\left(\hat{c}_{\mathbf{k} 1 \uparrow}^{\dagger} \hat{c}_{-\mathbf{k}+\mathbf{Q} 2 \downarrow}^{\dagger}-\hat{c}_{\mathbf{k} 1 \downarrow}^{\dagger} \hat{c}_{-\mathbf{k}+\mathbf{Q} 2 \uparrow}^{\dagger}\right)$.

In such case, the gap parameter is defined as follows:

$\Delta_{\mathbf{k} \mathbf{Q}}^{s}=-\frac{2}{\sqrt{2} N} \sum_{\mathbf{k}^{\prime}} J_{\mathbf{k}-\mathbf{k}^{\prime}}\left\langle\hat{B}_{\mathbf{k}^{\prime} m \mathbf{Q}}\right\rangle$.

In the absence of magnetic ordering, the self-consistent equations corresponding to both spin-triplet paired phase of type A and spin-singlet paired phase (with nonzero $\Delta_{\mathbf{k Q}}^{S}$ ) have the same form. Moreover, the free energies of those two phases are equal. In effect, the results presented in the next section are valid for both situations. This is caused by the fact that for the considered phases, the spin and band indices are treated on equal footing so the spin-triplet bandsinglet situation is equivalent to that with the spin-singlet band-triplet.

\section{Results and Discussion}

In our study, we consider the following phases: the normal phase (NS) with $\Delta_{\mathbf{Q}}=0$, the homogeneous superconducting phase $(S C-A)$ with $\Delta_{\mathbf{Q}} \neq 0, \mathbf{Q} \equiv 0$, and the inhomogeneous superconducting phase $(F F-A)$ with $\Delta_{\mathbf{Q}} \neq$ $0, \mathbf{Q} \neq 0$.

In the subsequent discussion, $n$ expresses the number of electrons per one $\mathrm{Fe}$ ion; the wave-vectors are given in the units of $1 / a$, where $a$ is the lattice parameter; and all the energies have been normalized to the bare bandwidth $W$, whereas $T$ represents the reduced temperature $T \equiv k_{\mathrm{B}} T / W$

One should note that our model with the interband pairing between the two Fermi surface sheets shown in Fig. 1a resembles the situation of one-band model with the spin-singlet pairing between the two spin subbands. The difference is that, here, the bottoms of the bands between which the pairing occurs coincide but the shape of the dispersion relations leads to Fermi wave-vector mismatch (c.f. Fig. 1a); whereas in the original idea of the FFLO phase, under the influence of the Zeeman term, the spin subbands are shifted as a whole. In our model, the mismatch can be tuned by changing $n$, because by increasing the Fermi level, one increases the distance between the Fermi sheets (c.f. Fig. 1).

We analyze first the gap symmetry given by (8) with two pairing components $\left(J_{0} \neq 0, J_{1} \neq 0\right)$. In Fig. 2 , we show that for the proper values of the band filling $n$, the freeenergy minima appear for nonzero values of the Cooper pair momentum $\mathbf{Q}$, which correspond to the stability of the FFA phase. As one can see in Fig. 2f, for $n=1.77514$, the free energy in the homogeneous paired phase (for $\mathbf{Q} \equiv 0$ ) is already greater than the free energy in the normal phase $(\Delta F>0)$. However, by setting the proper value of $\mathbf{Q}$, the stability of the superconducting phase can still be obtained. For the chosen gap symmetry, it is possible to connect the largest parts of the Fermi surfaces when $\mathbf{Q}$ is parallel to either the $k_{x}$ - or $k_{y}$-axis. In the FF-A phase, the population imbalance between the two bands occurs. As a result, some of the particles from the second band $(l=2)$ are not paired. The region in the reciprocal space which is occupied by the unpaired particles is shown in Fig. 3b. The corresponding quasiparticle dispersion relations in the FF-A phase are plotted in Fig. 3a.

In Fig. 4a, b, we show the stability regions of the considered phases in the $\left(n, J_{0}\right)$ space together with the values of the gap amplitudes. As one can see, the behavior of $\Delta^{(0)}$ and $\Delta^{(1)}$ is very similar except that the values of $\Delta^{(1)}$ are 1 order of magnitude smaller than those of $\Delta^{(0)}$. The critical temperature is the same for both, as it should be. The border between the stability regions of the SC-A phase and the NS phase for the case of no-FF-A phase included is marked by the solid line. One can see that the nonzero values of the Cooper pair momentum allows for the paired phase to adapt to the unfavorable conditions of the system with large Fermi wave-vector mismatch (the larger the band filling, the larger the mismatch). In effect, the region of stability of the paired phase is broadened by the FF-A phase. The transition from SC-A to FF-A phase has a discontinuous nature as the drop in both $\Delta^{(0)}$ and $\Delta^{(1)}$ occurs along the transition line. Additionally, in Fig. 4c, we show the free-energy difference between the inhomogeneous paired phase and the next lowest free-energy phase as a function of $n$ and $J_{0}$. The values of $Q_{x}$ which correspond to the free-energy minimum (FF-A phase stability) are provided in Fig. $4 \mathrm{~b}$.

According to our analysis, the FF-A phase can also be stable for the case of extended s-wave gap symmetry without the admixture of s-wave $\left(J_{0}=0, J_{1} \neq 0\right)$. The corresponding phase diagram on the $(T, n)$ plane is presented in Fig. 5. Here, also the discontinuous nature of the SC-A $\rightarrow$ FF-A transition is seen (the sudden drop of $\Delta^{(1)}$ in Fig. 5a). For the sake of completeness, we have made calculations for the d-wave gap symmetry given by (9). However, the $J_{1}$ parameter has to be quite large to obtain a stable dwave paired solution. In Fig. 6, we show that also in the last case, the free-energy minimum can appear for nonzero 
Fig. 2 Free energy in the paired state as a function of $\mathbf{Q}$ for the three values of the band filling: a $n=1.75$, b $n=1.76709$, and c $n=177514$. The pairing strength is set to $J_{0}=0.371992$ and $J_{1}=J_{0} / 5$. In $\mathbf{d}-\mathbf{f}$, the difference between the free energy in the paired and the normal phases $(\Delta F)$ is shown as a function of $Q_{x}$ for $Q_{y}=0$. The values of $n$ chosen for $\mathbf{d}-\mathbf{f}$ correspond to those from $\mathbf{a}-\mathbf{c}$, respectively

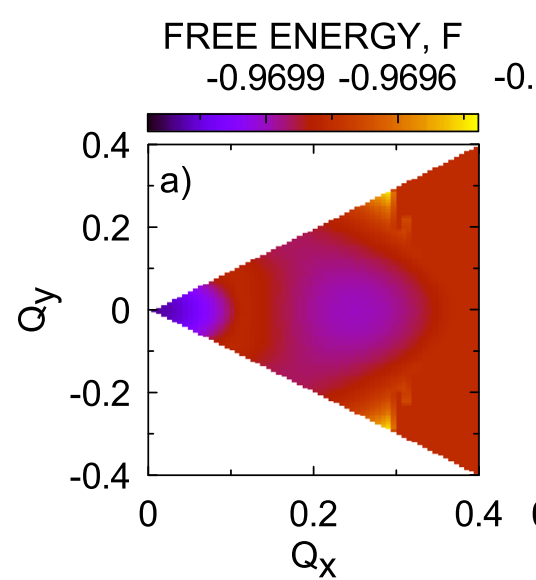

FREE ENERGY, $F$ $0.966-0.96595$

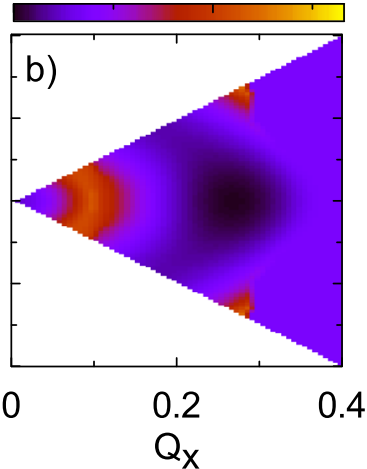

FREE ENERGY, F $-0.9645 \quad-0.9642$
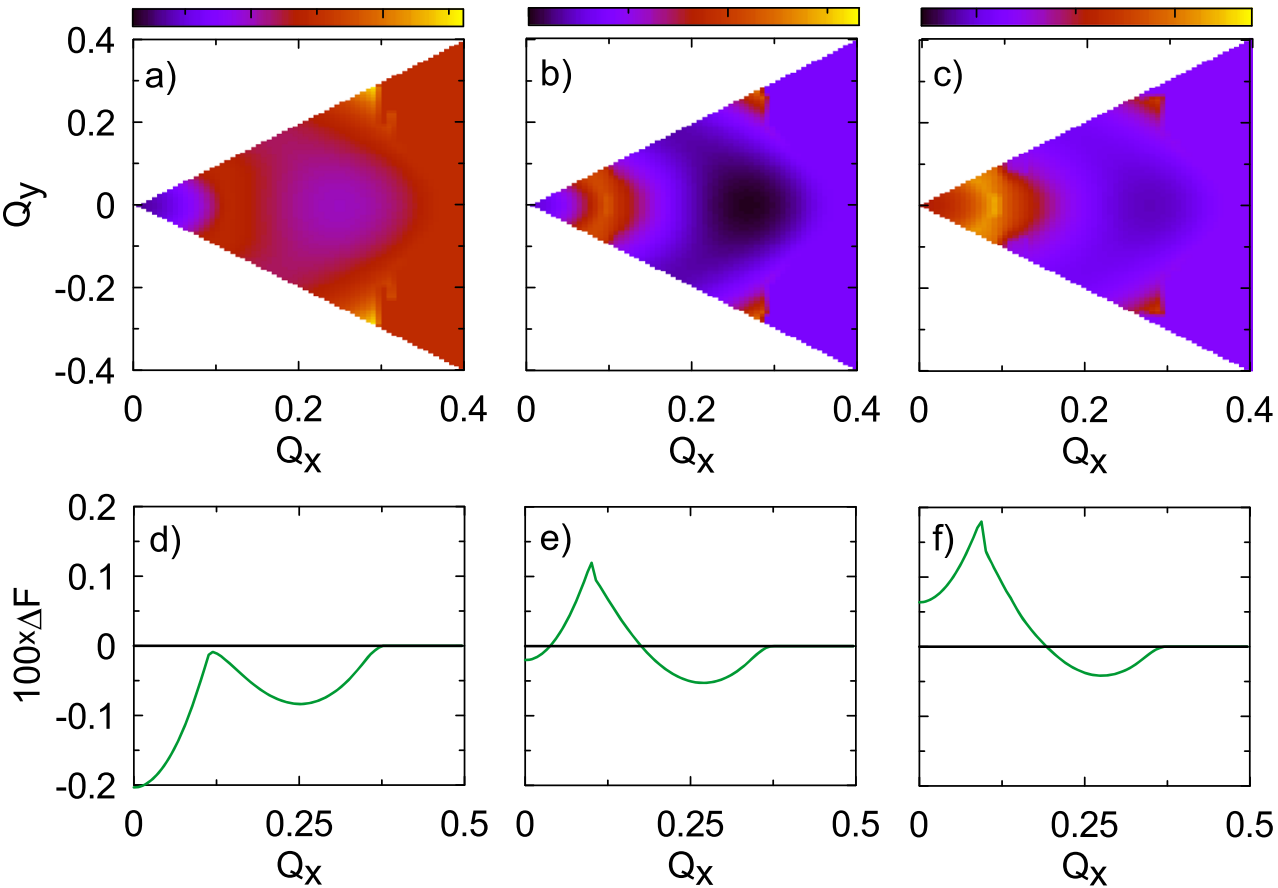

values of $\mathbf{Q}$. As one can see, the choice of the pairing symmetry influences the direction of the $\mathbf{Q}$ vector for which the free-energy minimum appears. In this case, the minimum is obtained for $Q_{x}=Q_{y}$ (and $Q_{x}=-Q_{y}$ ) direction, whereas for the case of s-wave symmetry (or the mixture of s-wave and extended s-wave symmetries) similar minimum is located on the $Q_{x}$ - or $Q_{y}$-axis (c.f. Fig. 2).

\section{Conclusions}

We have analyzed the possibility of a new kind of superconducting phase with a spontaneous nonzero Cooper pair momentum. This phase can occur without the external magnetic field in systems with the dominant interband pairing and two distinct Fermi surface sheets. The corresponding Fermi wave-vector mismatch which appears in such situation can be compensated by nonzero center-ofmass momentum of the Cooper pairs. In our study, we use as an example a tight binding model which describes the two hole-like bands of the iron-based superconductor $\mathrm{LaFeAsO}_{1-x} \mathrm{~F}_{x}$. The calculations have been carried out for different even-parity gap symmetries (s-wave, extended swave, and d-wave). We have shown that for proper values of the band filling and of the pairing strength, the free-energy minima appear which correspond to nonzero Cooper pair momentum. The direction of the $\mathbf{Q}$ vector depends on the selected gap symmetry (c.f. Figs. 2 and 6). For the case of the d-wave symmetry, the values of the pairing strength $J_{1}$ have to be very large $\left(J_{1}>1\right)$ to obtain a paired solution in the considered model.

In our approach, we use the mean field (BCS) approximation which overestimates both the values of the order parameters and the critical temperature, so it would be
Fig. 3 The quasiparticle dispersion relations in the FF-A phase: $\mathbf{a}$ for $n=1.798, J_{0}=0.3913$, and $J_{1}=J_{0} / 5$ along the trajectory in the folded Brillouin zone marked in $\mathbf{b}$ by the dashed line. The so-called depairing region for the same model parameters is shown in $\mathbf{b}$
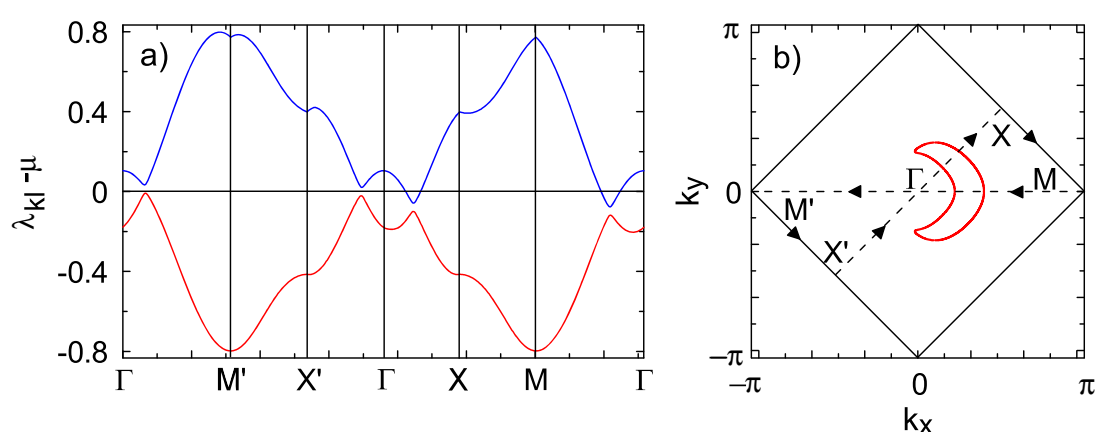
Fig. 4 The phase diagram in the $\left(n, J_{0}\right)$ space together with the values of $\Delta^{(0)}(\mathbf{a})$ and $\Delta^{(1)}(\mathbf{b})$ gap amplitudes. The solid line in $\mathbf{a}$ and $\mathbf{b}$ marks the stability border between the SC-A and NS phases for the case of no-FF-A phase included. $\mathbf{c}$ The free-energy difference between the FF-A phase and the next lowest free-energy phase $(\Delta F)$. d Values of the $Q_{x}$ component for $Q_{y}=0$, which minimize the free energy and lead to FF-A phase stability. For all points of the diagram, we set $J_{1}=J_{0} / 5$

Fig. 5 The phase diagram in the $(T, n)$ space a for $J_{1}=0.4836$ and $J_{0}=0$. b Values of the $Q_{x}$ component of the Cooper pair momentum (for $Q_{y}=0$ ) which correspond to FF-A stability

Fig. 6 Free energy of the paired phase as a function of the Cooper pair momentum for the case of d-wave gap symmetry with the pairing strength $J_{0}=0$ and $J_{1}=1.2066$ and $n=1.5$ a)
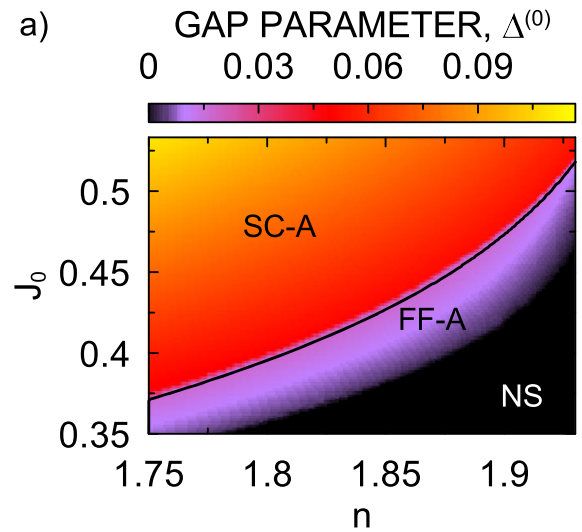

c)

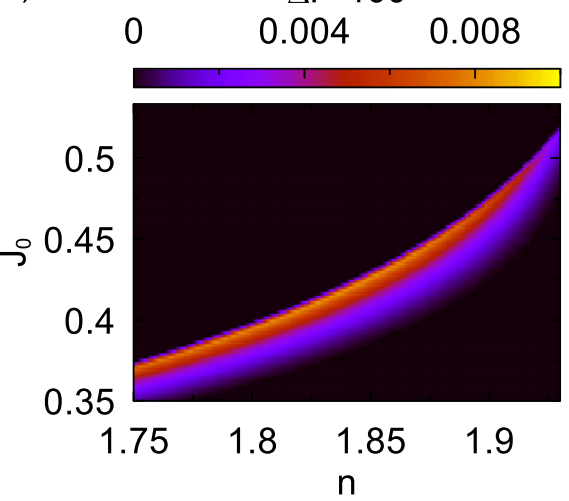

a)
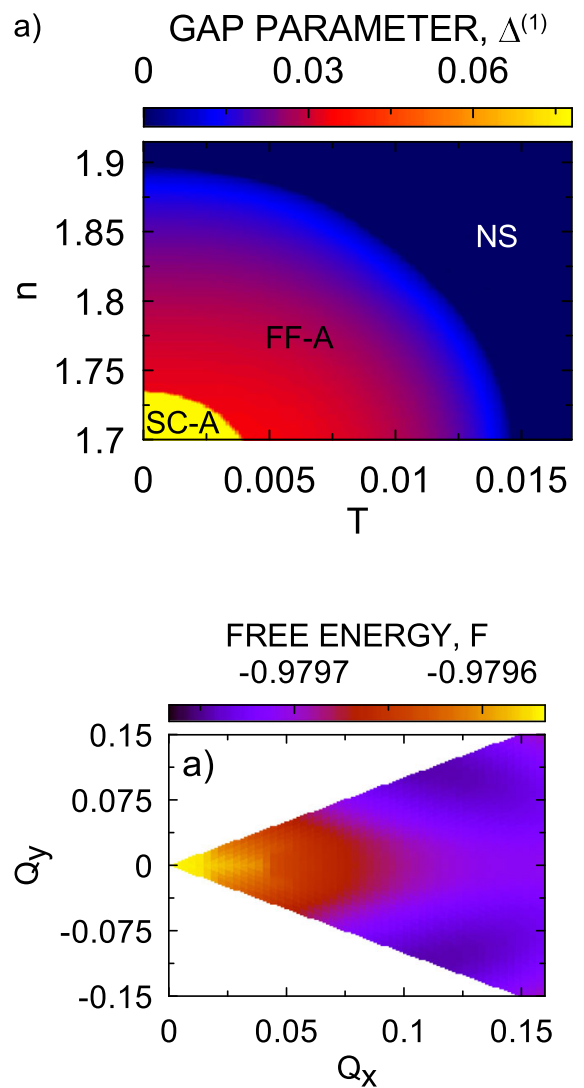

b) GAP PARAMETER, $\Delta^{(1)}$

$\begin{array}{llll}0 & 0.004 & 0.008 & 0.012\end{array}$

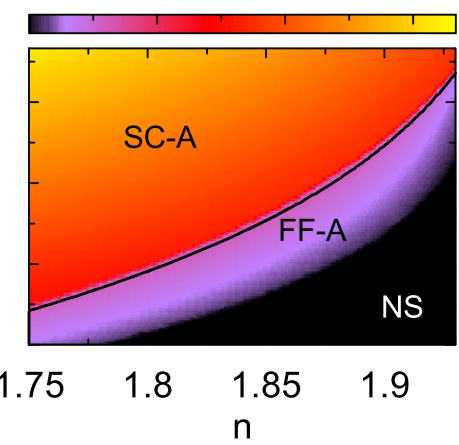

d) $\quad Q_{x}$

$\begin{array}{llll}0 & 0.2 & 0.4 & 0.6\end{array}$

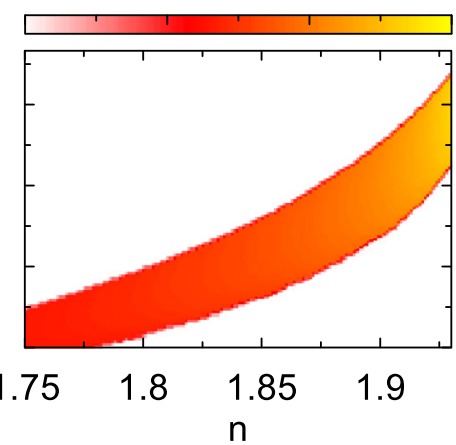

b) $\quad Q_{x}$ $\begin{array}{lllll}0 & 0.1 & 0.2 & 0.3 & 0.4\end{array}$
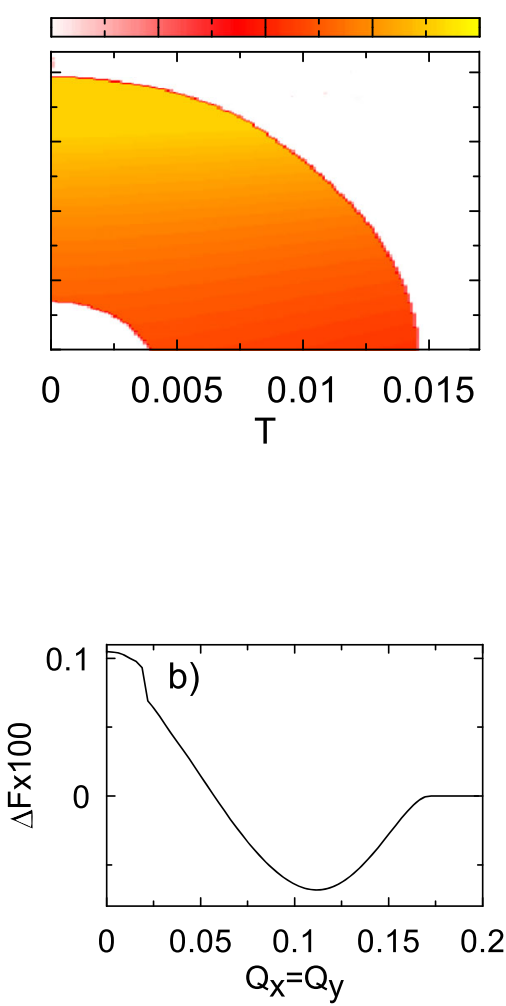
interesting to analyze the considered problem with the inclusion of interelectronic correlations. The spin-triplet interband pairing induced by the combined effect of Hund's rule and the correlations have been analyzed by us recently within the Gutzwiller approximation but without the possibility of a nonzero momentum pairing [22, 23]. Also, application of the proposed idea to other systems with the interband pairing seems reasonable. Namely, pairing between two species of particles with different (effective) masses could lead to a similar Fermi wave-vector mismatch as that considered above. Such an unconventional phase could be realized in systems of ultracold atomic gases in optical lattices. Spin-singlet pairing between particles with different effective masses has been theoretically investigated in $[24,25]$. However, in these considerations, the so-called spin-dependent masses are induced by interelectronic correlations and appear in an external magnetic field. As a result, the appearance of the nonzero momentum pairing is both due to the energy shift of the spin subbands and the corresponding modification of the dispersion relations due to spin-dependent renormalization factors.

As we have mentioned, the pairing induced by Hund's rule has an interband character. However, when it comes to other mechanisms, both inter- and intra-band components of the pairing can appear. The former can lead to the non-zero momentum of the Cooper pairs, whereas when the latter is strong, the homogeneous superconducting phase should be favored. It would be interesting to see to what extent the energy gain coming from the nonzero momentum pairing can survive in a model with both inter- and intra-band pairing. Another issue which would require further studies is the appearance of the degeneracy of the spin-triplet and spin-singlet pairings within our approach. This degeneracy should be broken by the spin-orbit coupling which has not been included by us at this stage of research. Moreover, the spin-orbit coupling would probably lead to a mixed ground state. These issues should be analyzed separately and are beyond the scope of this paper.

Acknowledgment The authors are grateful to the Foundation for Polish Science (FNP) for the support within the project TEAM, as well as to the National Science Center (NCN) through the Grant MAESTRO, No. DEC-2012/04/A/ST3/00342.
Open Access This article is distributed under the terms of the Creative Commons Attribution License which permits any use, distribution, and reproduction in any medium, provided the original author(s) and the source are credited.

\section{References}

1. Fulde, P., Ferrell, R.A.: Phys. Rev. 135, A550 (1964)

2. Larkin, A.I., Ovchinnikov, Y.N.: Sov. Phys. JETP 20, 762 (1964)

3. Saint-James, D., Sarma, G., Thomas, E.J.: Type II Superconductivity. Pergamon, New York (1969)

4. Bianchi, A., Movshovich, R., Cpan, C., Pagliuso, P.G., Sarrao, J.L.: Phys. Rev. Lett. 91, 187004 (2003)

5. Kumagai, K., Saitoh, M., Oyaizu, T., Furukawa, Y., Takashima, S., Nohara, M., Takagi, H., Matsuda, Y.: Phys. Rev. Lett. 97, 227002 (2006)

6. Correa, V.F., Murphy, T.P., Martin, C., Purcell, K.M., Palm, E.C., Schmiedenshoff, G.M., Cooley, J.C., Tozer, W.: Phys. Rev. Lett. 98, 087001 (2007)

7. Lee, I.J., Naughton, M.J., Danner, G.M., Chaikin, P.M.: Phys. Rev. Lett. 78, 3555 (1997)

8. Singleton, J., Symington, J.A., Nam, M.-S., Ardavan, A., Kurmoo, M., Day, P.: J. Phys. Condens. Matter. 12, L641 (2000)

9. Tanatar, M.A., Ishiguro, T., Tanaka, H., Kobayashi, H.: Phys. Rev. B 66, 134503 (2002)

10. Uji, S., Terashima, T., Nishimura, M., Takahide, Y., Konoike, T., Enomoto, K., Cui, H., Kobayashi, H., Kobayashi, A., Tanaka, H., Takumoto, M., Choi, E.S., Tokumoto, T., Graf, D., Brooks, J.S.: Phys. Rev. Lett. 97, 157001 (2006)

11. Shinagawa, J., Kurosaki, Y., Zhang, F., Parker, C., Brown, S.E., Jérome, D., Christensen, J.B., Bechgaard, K.: Phys. Rev. Lett. (2007)

12. Liao, Y., Rittner, A.S.C., Paprotta, T., Li, W., Partridge, G.B., Hulet, R.G., Baur, S.K., Mueller, E.J.: Nature 467, 567 (2010)

13. Spałek, J.: Phys. Rev. B 63, 104513 (2001)

14. Zegrodnik, M., Spałek, J.: Phys. Rev. B 86, 014505 (2012)

15. Dai, X., Fang, Z., Zhou, Y., Zhang, F.C.: Phys. Rev. Lett. 101, $057008(2008)$

16. Mazin, I.I., Singh, D.J., Johannes, M.D., Du, M.H.: Phys. Rev. Lett. 101, 57003 (2008)

17. Kuroki, K., Onari, S., Arita, R., Usui, H., Tanaka, Y., Kontani, H., Aoki, H.: Phys. Rev. Lett. 101, 087004 (2008)

18. Chen, W.Q., Zhou, K.Y., Zhang, F.C.: Phys. Rev. Lett. 102, 047006 (2009)

19. Maier, T.A., Scalpino, D.J.: Phys. Rev. B 78m, 020514(R) (2008)

20. Lee, P.A., Wen, X.G.: Phys. Rev. B 78, 144517 (2008)

21. Raghu, S., Qi, X.L., Liu, C.X., Scalpino, D.J., Zhang, S.C.: Phys. Rev. B 77, 220503(R) (2008)

22. Zegrodnik, M., Spałek, J., Bünemann, J.: New J. Phys. 15, 073050 (2013)

23. Zegrodnik, M., Bünemann, J., Spałek, J.: New J. Phys. 16, 033001 (2014)

24. Kaczmarczyk, J., Spałek, J.: J. Phys. Condens. Matter 22, 355702 (2010)

25. Maśka, M.M., Mierzejewski, M., Kaczmarczyk, J., Spałek, J.: Phys. Rev. B 82, 054509 (2010) 